\title{
Abnormal cerebellar development and axonal decussation due to mutations in AHI1 in Joubert syndrome
}

\author{
Russell J Ferland ${ }^{1,10}$, Wafaa Eyaid ${ }^{2,10}$, Randall V Collura ${ }^{3}$, Laura D Tully ${ }^{1}$, R Sean Hill ${ }^{1}$, Doha Al-Nouri ${ }^{2}$, \\ Ahmed Al-Rumayyan ${ }^{4}$, Meral Topcu ${ }^{5}$, Generoso Gascon ${ }^{6,7}$, Adria Bodell ${ }^{1}$, Yin Yao Shugart ${ }^{8}$, \\ Maryellen Ruvolo ${ }^{3} \&$ Christopher A Walsh ${ }^{1,9}$
}

Joubert syndrome is a congenital brain malformation of the cerebellar vermis and brainstem with abnormalities of axonal decussation (crossing in the brain) affecting the corticospinal tract and superior cerebellar peduncles ${ }^{1-9}$. Individuals with Joubert syndrome have motor and behavioral abnormalities, including an inability to walk due to severe clumsiness and 'mirror' movements, and cognitive and behavioral disturbances ${ }^{5-8,10-12}$. Here we identified a locus associated with Joubert syndrome, JBTS3, on chromosome 6q23.2-q23.3 and found three deleterious mutations in $A H I 1$, the first gene to be associated with Joubert syndrome. AHI1 is most highly expressed in brain, particularly in neurons that give rise to the crossing axons of the corticospinal tract and superior cerebellar peduncles. Comparative genetic analysis of $\boldsymbol{A H I 1}$ indicates that it has undergone positive evolutionary selection along the human lineage. Therefore, changes in AHI1 may have been important in the evolution of human-specific motor behaviors.

oubert syndrome is an autosomal recessive brain disorder. Affected individuals show weakness, abnormal breathing and eye movements, clumsiness, cognitive difficulties and autistic behaviors ${ }^{5-7,10-13}$. Joubert syndrome is defined by the absence of the cerebellar vermis (Fig. 1e,f) and by the 'molar tooth sign', formed by an abnormal configuration of the superior cerebellar peduncles (SCPs) that connect the cerebellum to the midbrain and thalamus (Fig. 1d $)^{1-3,5-7,9-12}$. Joubert syndrome is defined by the absence of the cerebellar vermis (compare normal MRI, Fig. 1a,b, with the MRI from an individual with Joubert syndrome, Fig. 1d,e) and by the 'molar tooth sign', formed by an abnormal configuration of the superior cerebellar peduncles (SCPs) that connect the cerebellum to the midbrain and thalamus (compare Fig. 1c with Fig. 1f) ) $^{1-3,5-7,9-12}$. Corticospinal fibers that normally decussate in the medulla also fail to cross the midline in Joubert syndrome ${ }^{1-3,9}$, causing mirror movements, in which both hands move synchronously instead of alternating, in affected individuals. In addition, individuals with Joubert syndrome often are unable to walk owing to severe clumsiness resulting from aberrant decussation and cerebellar malformations. The midline crossing defects in Joubert syndrome are not universal, however, as the optic chiasm, corpus callosum and other decussating pathways are spared.

Joubert syndrome can be subdivided according to clinical and genetic findings. Classic Joubert syndrome (or Joubert syndrome type A; as described above) involves the brain but not the kidney or retina and previously has been linked to chromosome 9q34.3 (refs. $14,15)$. In addition, a new type of Joubert syndrome, Joubert syndrome type B or cerebello-oculo-renal syndrome, includes brain, renal and retinal abnormalities and is linked to chromosome 11p12-q13.3 (refs. 16,17). Given the potential for genetic heterogeneity in Joubert syndrome, we carried out a genome-wide screen at a resolution of $10 \mathrm{cM}$ and fine-mapping on three Saudi Arabian pedigrees from the same geographical region (pedigrees 1,2 and 3) with autosomal recessive Joubert syndrome. Because the affected individuals in these pedigrees had very similar radiographic features, we assumed that they had an allelic disorder. Using this approach, we defined a locus, JBTS3, on chromosome 6q23.2-q23.3 (between microsatellites D6S976 and D6S1009) that was identical by descent in all affected members of each of the three pedigrees (Fig. 2 and Supplementary Fig. 1 online). We then identified an additional pedigree from another region of Saudi Arabia (pedigree 4) that also showed linkage of Joubert syndrome to JBTS3 (Supplementary Fig. 1 online). We finally identified another pedigree from Turkey (pedigree 5) that mapped to JBTS3 and further narrowed the region of shared homozygosity (Fig. 2). We pooled the linkage data and lod scores for the five pedigrees, which resulted in a summed multipoint lod score of 8.36 (Fig. 3a) and a maximum

\footnotetext{
${ }^{1}$ Division of Neurogenetics and Howard Hughes Medical Institute, Department of Neurology, Beth Israel Deaconess Medical Center, Harvard Medical School, NRB 266 77 Avenue Louis Pasteur, Boston, Massachusetts 02115, USA. ${ }^{2}$ Department of Pediatrics, King Fahad National Guard Hospital, Riyadh 11426 , Kingdom of Saudi Arabia. ${ }^{3}$ Department of Anthropology, Harvard University, Cambridge, Massachusetts 02138, USA. ${ }^{4}$ Department of Neurology, King Fahad National Guard Hospital, Riyadh 11426, Kingdom of Saudi Arabia. ${ }^{5}$ Hacettepe University Faculty of Medicine, Department of Pediatrics, Section of Child Neurology, Ankara 06100, Turkey. ${ }^{6}$ Department of Neurosciences, King Faisal Specialist Hospital and Research Centre, Jeddah 21499 , Kingdom of Saudi Arabia. ${ }^{7}$ Clinical Neurosciences \& Pediatrics, Brown University School of Medicine, Providence, Rhode Island 02908, USA. ${ }^{8}$ Department of Epidemiology, Johns Hopkins Bloomberg School of Public Health, Baltimore, Maryland 21205, USA. ${ }^{9}$ Program in Biological and Biomedical Sciences, Harvard Medical School, Boston, Massachusetts 02115, USA. ${ }^{10}$ These authors contributed equally to this work. Correspondence should be addressed to C.A.W. (cwalsh@bidmc.harvard.edu).
}

Published online 22 August 2004; doi:10.1038/ng1419 
Figure $1 \mathrm{MRIS}$ of a normal brain and of the brain of an individual with Joubert syndrome and mutations in AHI1. MRIs are presented in an axial plane (a,d, the upper pons), in a coronal plane (b,e, the cerebellum and posterior cerebral cortex) and in a sagittal plane (c,f, a midsagittal section through the brain showing the cerebral cortex, cerebellum, midbrain and pons). Axial (a), coronal (b) and midsagittal (c) T1-weighted images from an unaffected child with a normal brainstem $(\mathbf{a}, \mathbf{c})$ and a normal cerebellar vermis . (a-c). Axial T1-weighted (d), coronal FLAIR (e) and midsagittal T1-weighted (f) images from a 2-y-old male child with Joubert syndrome and mutations in AHII from pedigree 3. The image shows the molar tooth sign (black arrowhead in

d) and the absence of the cerebellar vermis (white arrows in e,f). In the axial images from the affected individual (d), the failure of the superior cerebellar peduncles to decussate is indicated by the presence of the peduncles traveling horizontal to the brainstem and not crossing the midline. The brain structure that is present in the midsagittal section (f) at the level of the cerebellum is not the vermis but the right lobe of the cerebellum, which is in the plane of the midsagittal section because of the absence of the vermis. Scale bars, $5 \mathrm{~cm}$. two-point lod score of 6.31 (at recombination fraction $=0$ ) at marker D6S1626 (Fig. 3b) ${ }^{18}$.

Sequencing of candidate genes in the minimal region of linkage (Fig. 3c) identified three independent mutations in the Abelson helper integration site gene (AHI1), which encodes a putative cytoplasmic adaptor protein containing a coiled-coil domain, seven WD40 repeats and one $\mathrm{SH} 3$ domain $^{19}$ (Fig. 3d). In pedigree 1, we identified the (TGA; amino acid substitution R351X), in all three affected individuals (Supplementary Fig. 2 online). In pedigree 2, we identified the mutation $1303 \mathrm{C} \rightarrow \mathrm{T}$, which produces a stop codon (TGA; amino acid substitution R435X) in exon 7 of AHI1 in both affected individuals (Supplementary Fig. 2 online). We also observed this mutation in the mRNA of one affected individual, but not in controls (Supplementary Fig. 2 online). Finally, in pedigree 3 , we identified the mutation $1328 \mathrm{~T} \rightarrow \mathrm{A}$ in exon 7 , which produces a nonconservative V443D amino acid substitution, resulting in a change from a hydrophobic to a polar or charged residue (Supplementary Fig. 2 online). In each pedigree, the mutations segregated with the disease and were not found in $>400$ chromosomes from unrelated control individuals of Arabic, European, African or Asian origin (data not shown), suggesting that these mutations are not single-nucleotide polymorphisms. To date, we have not identified coding region mutations in pedigrees 4 and 5, suggesting that they have mutations either in noncoding regions of $A H I 1$ or in other, unidentified genes. These data suggest that mutations in AHI1 cause Joubert syndrome.

In human fetal tissue, AHI1 mRNA is highly expressed in brain and kidney and weakly expressed in liver and lung (as assessed by northern-blot analysis; data not shown). In adult human brain tissue, AHI1 mRNA is highly expressed in cerebellum and cerebral cortex and weakly expressed in the putamen (as assessed by northern-blot analysis; data not shown). We detected mouse Ahil mRNA in whole
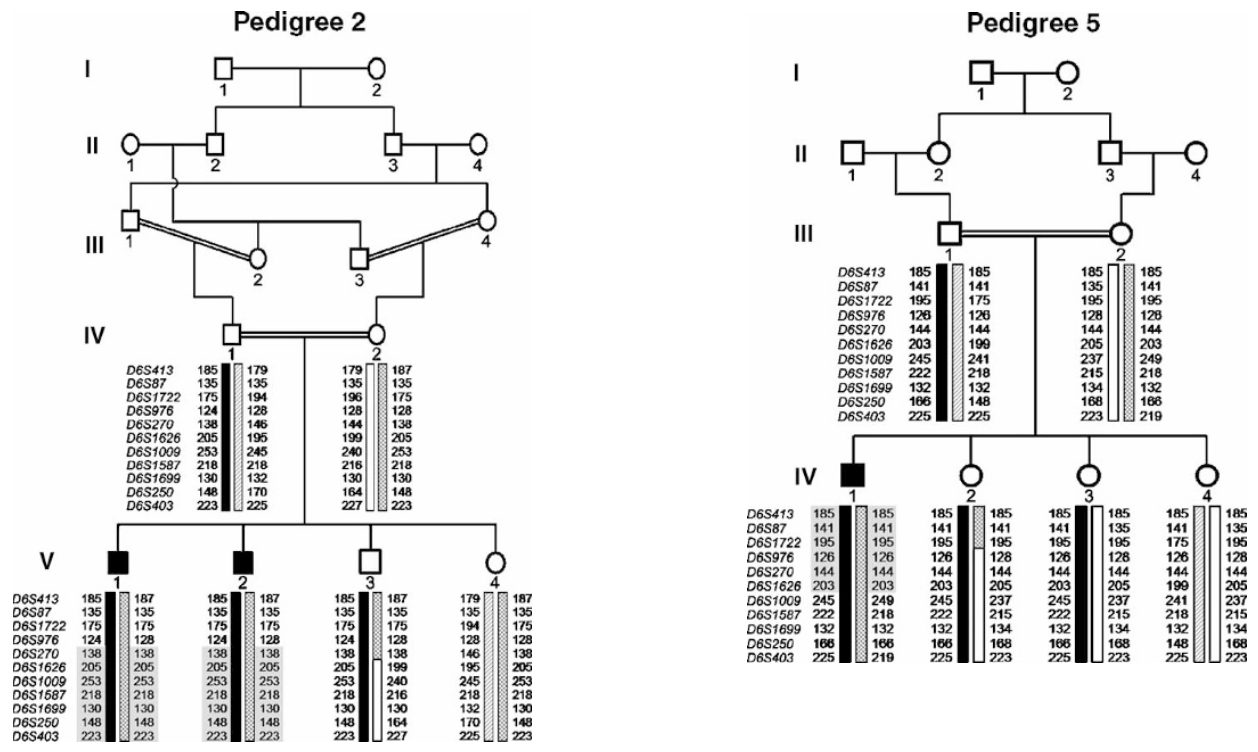

Figure 2 The genotypes of two (of five) pedigrees with Joubert syndrome that defined the smallest region of homozygosity. Pedigree 2 comes from Saudi Arabia and pedigree 5 comes from Turkey. The microsatellite markers that are presented are on chromosome 6q23.2-q24.1. The marker order is cenD6S413-D6S403-tel. The shaded regions of the allele lengths indicate the homozygous regions for each affected individual. The crossover regions are indicated in the columns adjacent to the alleles. Markers D6S976 and D6S1009 are not identical by descent in pedigree 2 and pedigree 5, respectively. 
a

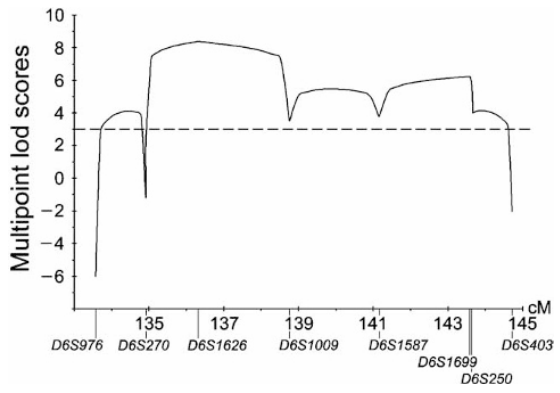

d

Figure 3 Linkage to JBTS3 and mutations in AHI1 in individuals with Joubert syndrome. (a) Combined multipoint lod score analyses of chromosome 6q23.2-q24.1 in the five pedigrees with Joubert syndrome generated a maximal multipoint lod score of 8.36 between microsatellite markers D6S270 and D6S1626. (b) Combined two-point lod score analyses in the five pedigrees with Joubert syndrome generated a maximal two-point lod score of 6.31 at microsatellite D6S1626. (c) Genomic segment on chromosome 6 corresponding to the JBTS3 candidate interval, including the relative positions of candidate genes in this region. (d) Schematic diagram of the structure of human AHI showing the main domains of this protein: the coiled-coil domain, the WD40 repeats and the SH3 domain ${ }^{19}$. The diagram also indicates the mutations that we identified in individuals with Joubert syndrome and their locations.

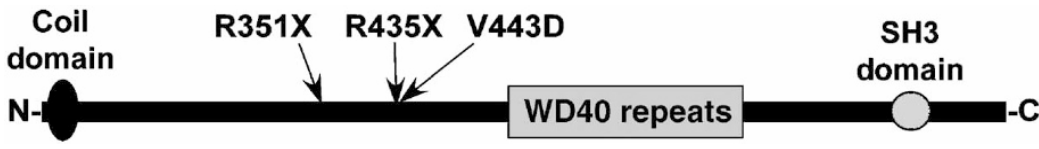

\begin{tabular}{lrrrrrrr} 
b Order & 0.0 & 0.01 & 0.05 & 0.1 & 0.2 & 0.3 & $(\theta)$ \\
\hline$D 6 S 413$ & - Infini & 1.36 & 2.24 & 2.21 & 1.62 & 0.93 & \\
$D 6 S 87$ & 4.76 & 4.64 & 4.13 & 3.51 & 2.32 & 1.29 & \\
$D 6 S 1722$ & - Infini & 3.19 & 3.33 & 2.95 & 1.96 & 1.03 & \\
$D 6 S 976$ & 2.20 & 3.67 & 3.81 & 3.42 & 2.39 & 1.38 & \\
$D 6 S 270$ & - Infini & 3.52 & 3.63 & 3.21 & 2.14 & 1.15 & \\
$D 6 S 1626$ & 6.31 & 6.14 & 5.45 & 4.60 & 2.99 & 1.60 & \\
$D 6 S 1009$ & 1.18 & 2.05 & 2.31 & 2.11 & 1.45 & 0.80 \\
$D 6 S 1587$ & 1.18 & 2.05 & 2.31 & 2.11 & 1.45 & 0.80 \\
$D 6 S 1699$ & 4.34 & 4.22 & 3.74 & 3.15 & 2.03 & 1.09 \\
$D 6 S 250$ & 5.44 & 5.29 & 4.66 & 3.89 & 2.45 & 1.26 & \\
$D 6 S 403$ & 3.33 & 3.89 & 3.87 & 3.37 & 2.17 & 1.08 &
\end{tabular}

embryos as early as embryonic day (E) 10.5 with continued expression during prenatal brain development (Supplementary Fig. 3 online). Expression decreased two weeks after birth and into adulthood (Supplementary Fig. 3 online). As assessed by in situ hybridization, mouse Ahil mRNA was expressed throughout the brain, most highly in the developing and mature hindbrain and spinal cord (from E14.5 to adulthood; Fig. 4). Ahil expression was weak in the cerebellum primordium at E12.5 (data not shown) and E16.5 (Fig. 4c), but peaked at E14.5 (Fig. 4a,b). Moreover, Ahil was highly expressed in the deep cerebellar nuclei and weakly expressed in the cerebellar (2) hemispheres from E16.5 to adulthood (Fig. 4c-f,h). Overall, the cerebellar expression pattern paralleled the cerebellar malformation associated with Joubert syndrome, which severely affects midline structures (cerebellar vermis and deep nuclei) and largely spares the laterally placed cerebellar hemispheres.

Ahil mRNA is expressed in the cell bodies that give rise to axonal tracts that fail to decussate at the midline in Joubert syndrome, such as the cell bodies of the cerebellar dentate nucleus (Fig. $4 \mathbf{4}, \mathbf{i}$ ) that send axons across the midline, forming the SCPs, and in the neuronal cell bodies of the deep layer V cerebral cortical neurons (Fig. $\mathbf{4 c - g}$ ) that give rise to the corticospinal tract that decussates in the medullary pyramids. Ahil mRNA expression in the oculomotor nerve nuclei may account for the abnormal eye movements in individuals with Joubert syndrome (data not shown). Our results suggest that AHI1 may be a crucial cell-autonomous modulator of axonal decussation, because it is expressed in neurons that give rise to some crossing pathways and because mutations in AHII prevent proper decussation in individuals with Joubert syndrome.

Ahil mRNA is also highly expressed in other brain regions that are not directly implicated in the Joubert syndrome phenotype, such as the cerebral cortex, hippocampus, basal ganglia and hypothalamus and weakly expressed in the thalamus (from E16.5 to adulthood; Fig. 4c-f). If the expression pattern in humans parallels that in mice, then some of the behavioral phenotypes associated with Joubert syndrome may be mediated by structures that are not usually malformed, such as the cerebral cortex.
The predicted human AHI1 protein contains a coiled-coil domain in its $\mathrm{N}$-terminal 140 amino acids ${ }^{19}$ (corresponding to nucleotides $1-420$ ). These amino acids and this domain are entirely missing in the predicted proteins of both mouse and rat ${ }^{19}$ but are present in the predicted proteins of nonhuman primates and other mammals (cow, pig, dog and cat). This indicates that the coiled-coil domain is not unique to primates and was probably lost in the rodent lineage (Supplementary Fig. 4 online). The differences in the AHIl sequence between species alerted us to the possibility that the portion of the gene encoding the $\mathrm{N}$ terminus might be particularly dynamic evolutionarily. We then aligned and compared the sequences of AHI1 proteins from various primate species and found that amino acid differences between the species were concentrated in the first half of the coding region (nucleotides 1-1,794), particularly in the coiled-coil domain.

We constructed a phylogenetic tree of hominoid AHI1 sequences and used it to test for evidence of natural selection. In molecular evolutionary terms, the degree of protein evolution relative to the 'silent' mutation rate is measured by the ratio of nonsynonymous (amino acid-altering) changes per nonsynonymous site $\left(K_{\mathrm{a}}\right)$ to synonymous (silent) changes per synonymous site $\left(K_{\mathrm{s}}\right)$; high ratios are consistent with directional selection ${ }^{20,21}$. The human lineage has a significantly higher $K_{\mathrm{a}} / K_{\mathrm{s}}$ value $(1.26 ; P<0.05)$ than the other branches of the AHI1 tree combined (Fig. 5). This pattern of selection was more pronounced when we analyzed only the $5^{\prime}$ half of the coding region, containing the coiled-coil domain $\left(K_{\mathrm{a}} / K_{\mathrm{s}}\right.$ value $=2.79 ; P<0.05$; Fig. 5). Only 2 of the 231 genes recently analyzed on chimpanzee chromosome 22 have a $K_{\mathrm{a}} / K_{\mathrm{s}}$ value greater than 2.79 (observed for the $5^{\prime}$ half of AHI1); 15 of the 231 genes have a $K_{\mathrm{a}} / K_{\mathrm{s}}$ value greater than 1.26 (for fulllength $A H I 1)^{22}$. Accelerated evolution of $A H I 1$ in the human lineage could indicate either the action of directional selection or relaxed functional constraint. Given that AHI1 has a crucial function in human brain development, as indicated by the Joubert syndrome phenotype, relaxed functional constraint is probably not the cause. 

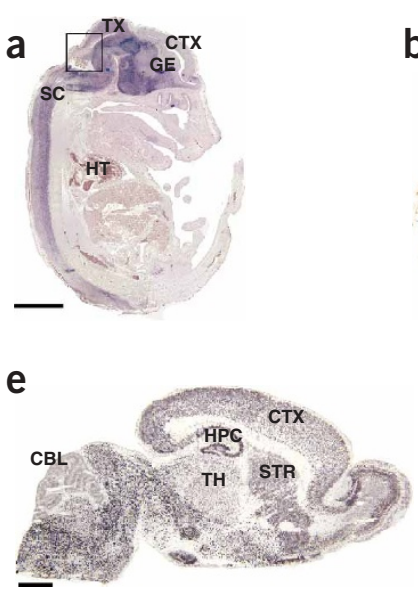

b

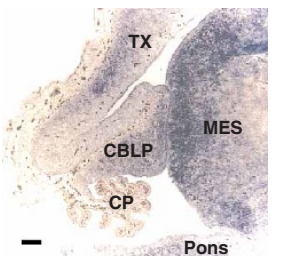

C

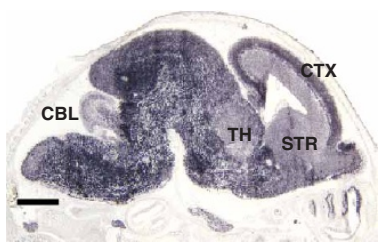

d

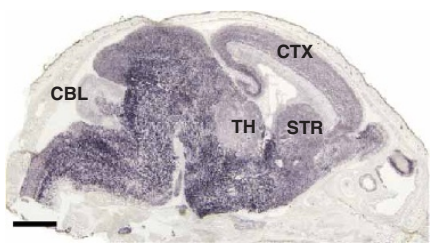

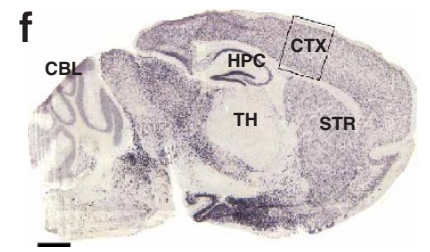

g
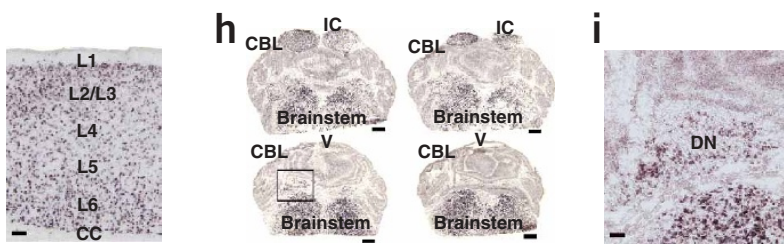

Figure 4 Spatial expression of mouse Ahi1 in brain during embryonic and postnatal development. In situ hybridizations for Ahi1 mRNA in sagittal sections of mouse brain at E14.5 (a,b), E16.5 (c), postnatal day (P) O (d), P6 (e,h,i) and P21 (f, g). Ahil mRNA is widely expressed throughout the brain, particularly in the hindbrain. Weaker expression occurs in the developing cerebellar primordium (E12.5 and E16.5), with the highest expression occurring at E14.5. Ahi1 is also expressed in the olfactory bulb, hippocampus (dentate gyrus, CA1-3), neocortex, basal ganglia and spinal cord. (h,i) Ahi1 mRNA expression in sequential sections through the cerebellum of a P6 mouse brain. Ahi1 is expressed relatively weakly in the cerebellar hemispheres after embryogenesis. Ahil expression is high in the cerebellar dentate nuclei, the output nucleus of the cerebellum that forms the superior cerebellar peduncle (h), and the deep layer cortical neurons (layer 5 ) that form the corticospinal tract (c-g). These patterns of expression (a-i) were indistinguishable using separate antisense probes. No specific staining was observed using sense probes (data not shown). Scale bars: a,c-f, $1 \mathrm{~mm} ; \mathbf{h}, 500 \mu \mathrm{m} ; \mathbf{b}, \mathbf{g}, \mathbf{i}, 100 \mu \mathrm{m}$. CBL, cerebellum; CBLP, cerebellar primordium; CC, corpus callosum; CP, choroid plexus; CTX, cerebral cortex; DN, dentate nucleus of the cerebellum; GE, ganglionic eminences; HPC, hippocampus; HT, heart; IC, inferior colliculus; L, layers of the cerebral cortex; MES, mesencephalon; SC, spinal cord; STR, striatum; TH, thalamus; TX, tectum; V, cerebellar vermis.

Using likelihood and parsimony methods, 12 amino acid changes in AHI1 were determined to have occurred in the human lineage ${ }^{20,21}$. To test whether these changes occurred early in hominid evolution, before the divergence of modern humans, we sequenced these sites in several diverse individuals. We found no substitutions altering these 12 amino acids, indicating that these changes occurred before the divergence of modern humans. Amino acid evolution was only approximately half (2) as fast on the chimpanzee branch (six reconstructed amino acid changes). The branch leading to the human-chimpanzee clade had an intermediate $K_{\mathrm{a}} / K_{\mathrm{s}}$ value (Fig. 5), indicating that selection on AHI1 may have begun before the divergence of these species but did not continue on the chimpanzee branch. Although they are not definitive, these results suggest that positive darwinian selection occurred in AHI1 in the direct ancestors of humans and has been particularly

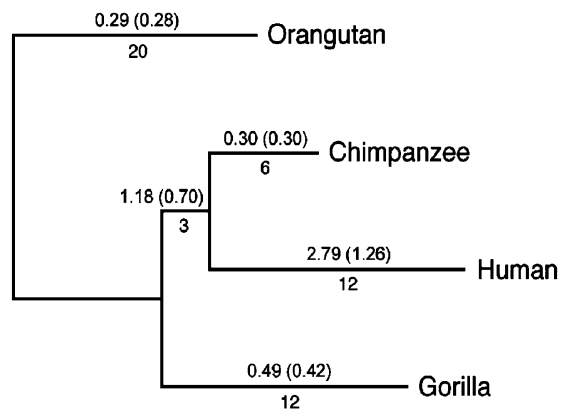

Figure 5 Phylogenetic tree showing AHI1 gene evolution in hominoids. Branch lengths are drawn proportional to the amount of protein change reconstructed along each branch by PAUP* (with numbers of amino acid changes shown below the branches). $K_{\mathrm{a}} / K_{\mathrm{s}}$ values (using the free-ratio model in the CODEML program of the PAML package ${ }^{20,21}$ ) are above the branches for the $5^{\prime}$ half of the gene $(1,794 \mathrm{bp})$ and in parentheses for the entire gene (3,588 bp, excluding the stop codon). pronounced in the first half of the gene. Key directional selection on AHI1 probably occurred after the common ancestor of human and chimpanzee, 6-7 million years ago, and before the common ancestor of modern humans, $\sim 200,000$ years ago.

Our studies identify the first gene associated with Joubert syndrome, $A H I 1$, and show that this gene is expressed in brain structures with neuroradiological abnormalities and in commissural neurons that fail to decussate (corticospinal tract and SCPs) in individuals with Joubert syndrome. Several molecules are crucial modulators of commissural fiber crossing, including Slits, Robo and Ephrins ${ }^{23,24}$, but it is not clear how these membrane complexes regulate downstream cytoplasmic signaling partners. AHI1 is as a potential downstream effector of these pathways, as it is essential for commissural crossing in the corticospinal tract and SCPs and encodes a putative cytoplasmic phosphoprotein. Determining the signaling pathways by which AHI1 modulates cellular signals may help elucidate potential genes underlying the other two loci known to be associated with Joubert syndrome (JBTS1 and CORS2, also called JBTS2) ${ }^{15-17}$, as it is reasonable to speculate that such genes may be in the AHI1 pathway. Although we have no direct evidence, it seems that the Joubert syndrome phenotype, with its aberrant axonal patterns and associated motor abnormalities, combined with the molecular evolutionary pattern of selection and the evolutionary increase in the corticospinal tract of primates ${ }^{25}$, potentially implicates $A H I 1$ in the evolution of some aspects of the distinctive motor programs that characterize humans.

\section{METHODS}

Subjects. All magnetic resonance images (MRIs; or computed tomography scans) of affected individuals were read by multiple neurologists and Joubert syndrome was diagnosed using previously described criteria ${ }^{2,9,26,27}$. Clinical features of children with Joubert syndrome include an inability to walk due to severe clumsiness, oculomotor abnormalities, hypotonia, ataxia, developmental delay with mental retardation, and mirror movements. None of the children 
diagnosed with Joubert syndrome that showed linkage to JBTS3 or was associated with mutations in AHI1 had any renal abnormalities.

Clinical features of the children with Joubert syndrome in pedigree 1 include nystagmus and ocular apraxia (both eye movement abnormalities), hypotonia (weakness), ataxia (clumsiness), developmental delay with mental retardation and mirror movements. In pedigree 2, all of the children with Joubert syndrome have nystagmus or ocular apraxia, hypotonia, ataxia, developmental delay with mental retardation and mirror movements (in one of the two affected children). The child with Joubert syndrome in pedigree 3 has hypotonia, is unable to walk and has developmental delay and mental of retardation (no information is available on the presence of eye or mirror movements). Moreover, the parents of this child had two previous children who had mental retardation and died early in infancy (no MRIs are available). In pedigree 4 , the one child with Joubert syndrome is hypotonic, unable to walk and mentally retarded and has a seizure disorder (no information is available on the presence of mirror movements). Finally, the child with Joubert syndrome in pedigree 5 has ptosis, nystagmus and exotropia (eye movement abnormality) in addition to ataxia, mental retardation and autism (no information is available on the presence of mirror movements).

The study was reviewed and approved by the Institutional Review Board at the Beth Israel Deaconess Medical Center and at Children's Hospital Boston in accordance with the National Institutes of Health. Informed consent was obtained from all of the subjects participating in this study. All animal use procedures were reviewed and approved by the Harvard Medical School Standing Committee on Animals in accordance with the National Institutes of Health.

Linkage analysis. We carried out genome-wide screens using fluorescently labeled human MapPairs separated at an average interval of $10 \mathrm{cM}$ (ABI o Linkage Mapping Set Version 2.5, Applied Biosystems) on DNA isolated from peripheral whole blood using a kit in accordance with the manufacturer's protocol (Qiagen). We carried out PCR using the various MapPair microsatellite markers and analyzed the products on an ABI Prism 3100 genetic analyzer. We determined all allele sizes using Genotyper 3.7 (Applied Biosystems) in conjunction with fluorescence size standards.

We further refined and narrowed the region of linkage using the following markers: D6S1698, D6S1656, D6S270 (ABI Linkage Mapping Set Version 2.5, Applied Biosystems) and D6S457, D6S413, D6S87, D6S1722, D6S976, D6S1626, D6S1009, D6S1587, D6S1699, D6S250, D6S403, D6S1675, D6S1684, D6S944, D6S453, D6S1648, D6S310, D6S958, D6S383 (custom synthesis; Research Genetics, Invitrogen).

Both parametric two-point and multipoint analyses assumed an autosomal recessive genetic model with complete penetrance in males and females and a frequency of the disease-associated allele of 0.001. Allele frequencies were assumed to be equal for all analyses. We carried out parametric multipoint linkage analysis using the method of location scores implemented in SIMWALK2 (version 2.86) ${ }^{28,29}$. As described previously ${ }^{28}$, location scores indicate the likelihood of several putative positions, among the marker loci, for the trait locus. These location scores are directly comparable with multipoint lod scores and are presented in $\log 10$ units. Results from the multipoint analyses are illustrated in Figure 3a. We carried out two-point parametric analyses using FASTLINK (version $4.1 \mathrm{p})^{30}$ assuming a range of $\theta$ values $(0.0-0.5)$. Results are summarized in Figure 3b. Analyses that assumed a variation in the penetrance of the disease (70-100\%) did not significantly alter the lod score analysis (data not shown).

Candidate gene analysis. We identified candidate genes in the region of homozygosity that was identical by descent using available genomic databases (University of Santa Cruz, National Center for Biotechnology Information (NCBI) and Celera). Based on the information in these databases (i.e., expression patterns, size of the gene, previous association with other disorders), we assessed the likelihood that mutations in certain candidate genes caused Joubert syndrome. There were 11 genes in the candidate interval that maps to the minimal identical-by-descent region (Fig. 3c). Before identifying mutations in $A H I 1$ in the individuals with Joubert syndrome that we studied, we sequenced four genes (SGK, ALDH8A1, HBS1L and MAP7) but did not identify any amino acid-changing mutations. Primers were designed to amplify each exon and its splice acceptor sites using Primer3 and were synthesized by Sigma-Geneosys. Primer sequences are available on request. We amplified exons by PCR and purified them using either the PSIYClone PCR 96 kit (Princeton Separations) or the AMPure kit (Agencourt) in accordance with the manufacturer's protocols. We sequenced all PCR products bidirectionally by fluorescent dye-terminator chemistry by Seqwright. We analyzed sequence data from each exon and its intron-exon boundary using standard analysis software for DNA sequencing (SeqMan II, version 5.03, DNASTAR) and compared them against the consensus sequences available from the University of California at Santa Cruz, NCBI and Celera. We sequenced normal control DNA samples obtained from the chromosomes $(>400)$ of random individuals of Arabic $(n=$ $80)$, European $(n=240)$, Chinese $(n=20)$, Japanese $(n=20)$, Southeast Asian (not from China or Japan, $n=20)$, African $(n=18)$ or Pygmy $(n=20)$ descent to look for mutations in exons 6 and 7 and found none (data not shown), suggesting that these mutations are not common polymorphisms. All control DNA samples were from the Coriell Human Variation Panels (Coriell Institute for Medical Research). Sample numbers are available on request. We also searched the SNP databases of both NCBI and Celera and found that these mutations were not variants in the general population.

Human RNA isolation. We obtained RNA from Epstein-Barr virus-transformed lymphocytes from one of the affected individuals in pedigree 2. We used reverse transcriptase reactions with both random hexamers and oligo dT primers to obtain CDNA. We carried out PCR and sequencing using primers designed to amplify the coding region for exon 7 of $A H I 1$ (primer sequences available on request).

Mouse in situ hybridization. We carried out nonradioactive in situ hybridization on frozen mouse brains, using digoxigenin-labeled cRNA probes. We generated the Ahil probes by PCR from IMAGE clone 6467369 (BC055400) at the following nucleotide sequence positions in the Ahil cDNA: Ahi1-1 (1-969; 969 bp), Ahi1-2 (652-1,623; 972 bp), Ahi1-3 (2,125-2,979; 855 bp) and Ahi1-4 (3,147-4,002; 856 bp; $3^{\prime}$ untranslated region). All Ahil nucleotide probe sequences were from nucleotides downstream of the ATG start site. All forward primers contained $\mathrm{T} 3$ promoter sequences on their $5^{\prime}$ ends to drive the transcription of the cRNA sense probes. All reverse primers contained T7 promoter sequences on their $5^{\prime}$ ends drive the transcription of the cRNA antisense probes. Primers were designed using Primer 3 and primer sequences are available on request.

All in situ hybridization studies were done using digoxigenin-labeled cRNA probes. We sectioned frozen brains $(10-20 \mu \mathrm{m})$ in a cryostat and positioned them onto Superfrost-plus microscope slides. We fixed the sections, acetylated them and hybridized them at $68{ }^{\circ} \mathrm{C}$ for three nights using each probe (approximate concentration of $100 \mathrm{ng} \mathrm{ml}^{-1}$ ). We visualized hybridized probes using alkaline phosphatase-conjugated anti-digoxigenin Fab fragments (Roche) and 5-bromo4-chloro-3-indolyl-phosphate-nitro blue tetrazolium substrate (Kierkegard and Perry Laboratories). We washed sections several times in $100 \mathrm{mM}$ Tris, $150 \mathrm{mM}$ $\mathrm{NaCl}$ and $20 \mathrm{mM}$ EDTA ( $\mathrm{pH}$ 9.5) and placed them on coverslips with glycerol gelatin (Sigma). All four Ahil antisense probes yielded comparable expression patterns. We incubated control sections either in an identical concentration of the sense probe transcript or with another Ahil probe directed against a different sequence from the same gene to determine whether this second probe had an identical labeling pattern. We examined the sense control probes and found that they gave no specific hybridization signal (data not shown).

Northern-blot analysis. We carried out northern-blot analysis of human AHI1 mRNA (fetal or adult) using commercially available mRNA filters obtained from Clontech Laboratories. We carried out northern-blot analysis of mouse RNA (embryonic whole embryos or mouse brain) using a commercially available RNA filter from Seegene. We used a northern-blot kit from Kierkegard and Perry Laboratories to prepare the probe and carry out hybridization and detection in accordance with the manufacturer's instructions. We generated the human AHI1 probes by PCR from IMAGE clones 4077654 (BC029417) and 3908210 (BC015019). We generated human northern-blot probes from PCR-amplified sequences at the following nucleotide sequence positions in the AHI1 cDNA: AHI1-1 (35-881; 847 bp; IMAGE 4077654) and AHI1-2 (1,641-2,723; 1,083 bp; IMAGE 3908210) using primers constructed to amplify these sequences in combination with the Detector PCR DNA Biotinylation Kit (Kierkegard and Perry Laboratories). All AHI1 probe sequences were from nucleotides 
downstream of the ATG start site in the AHI1 coding region. Primers were designed using Primer3 and primer sequences are available on request.

We used the same probes (Ahi1-3 and Ahi1-4) that were used for the mouse in situ hybridization experiments in the northern-blot analysis, except that we used the Detector PCR DNA Biotinylation Kit (Kierkegard and Perry Laboratories) to label the mouse probes. We used the same northern-blot kit (Kierkegard and Perry Laboratories) for the mouse filters that we used for the human filters. We used ethidium bromide staining to assess equal loading of the lanes.

Phylogenetic analysis. We obtained genomic DNA from a diverse selection of humans throughout world populations (Homo sapiens; Coriell Human Variation Panels, Coriell Institute for Medical Research) including two individuals from Africa (South of the Sahara; NA17345 and NA17344), two individuals from Japan (NA17051 and NA17052), two individuals from China (NA17016 and NA 17017), two individuals from Southeast Asia (excluding China and Japan; NA17085 and NA 17084) and two individuals from the Pygmy population of Africa (NA10492 and NA10493). The PCR conditions and sequencing of AHI1 were the same as those used for the other human individuals sequenced in our study.

We obtained genomic DNA from nonhuman primates from The Yerkes Regional Primate Research Center: common chimpanzee (Pan troglodytes; YB84-254, male), lowland gorilla (Gorilla gorilla; YN90-47, male) and Sumatran orangutan (Pongo pygmaeus; YB84-286, male). We used human AHI1 primers, described above, to amplify each exon by PCR for each of these species, with occasional variation of the PCR annealing temperatures. We purified PCR products as described above and sequenced them bidirectionally by fluorescent dye-terminator chemistry (Seqwright). We constructed sequence data from each of the exons and combined them for each species to generate complete coding region DNA sequences, using standard analysis software for DNA sequencing (SeqMan II).

We carried out tests of positive selection in CODon Maximum Likelihood (codeml) in PAML v.3.13 (ref. 20). The methodology of likelihood ratio tests is described elsewhere ${ }^{21}$. Trees and branch lengths were calculated in PAUP ${ }^{\star}$ (PAUP v.4.0b10).

The following reconstructed amino acid changes in AHI1 occurred in the human lineage: K39R, M94T, S106L, K148N, N180T, K206R, E385G, M455V, Q508P, N510S, K692R and V777I.

To calculate the percentage differences in amino acid composition between human and mouse, orangutan, gorilla or chimpanzee, we used the program (2) RevTrans 1.0 to align all the sequences. We used MEGA2 to determine the differences in amino acids between the various species. We calculated percent differences by determining the number of differences between species and dividing this value by the appropriate number of total amino acids. For domain analyses of the percent differences, we used domain structures from ref. 19 and independently verified the domains using the conserved domain database at NCBI.

URLs. The mouse and human genome databases from the University of California at Santa Cruz, NCBI and Celera are available at http://genome.ucsc. edu/, http://www.ncbi.nlm.nih.gov/ and http://www.celera.com/, respectively. Primer3 is available at http://frodo.wi.mit.edu/cgi-bin/primer3/primer3_ www.cgi. RevTrans 1.0 is available at http://www.cbs.dtu.dk/services/RevTrans/ and MEGA2 is available at http://www.megasoftware.net/.

Accession numbers. GenBank: human AHI1 genomic DNA, NT_025741; human AHI1 mRNA, NM_017651; mouse Ahil genomic DNA, NT_039491; mouse Ahil mRNA, NM_026203. GenBank protein: human AHI1 protein, NP_060121; mouse Ahil protein, NP_080479.

Note: Supplementary information is available on the Nature Genetics website.

\section{ACKNOWLEDGMENTS}

We thank D. Reich, D. Pilbeam and members of the laboratory of C.A.W. for discussions; U. Berger and C. Patoine for help with the in situ hybridization studies; the Yerkes Regional Primate Research Center for primate tissues; and the families who participated in these studies. This work was supported by grants (to C.A.W.) from the National Institute of Neurological Disorders and Stroke, the March of Dimes, and the McKnight Foundation. C.A.W. is an Investigator of the Howard Hughes Medical Institute. R.J.F. is supported by a Young Investigator Award from Cure Autism Now. This work was also supported by a Mind/Brain/
Behavior Interfaculty Initiative grant to M.R. and C.A.W. R.V.C. was supported by a Jacob K. Javits Graduate Fellowship.

\section{COMPETING INTERESTS STATEMENT}

The authors declare that they have no competing financial interests.

Received 4 June; accepted 4 August 2004

Published online at http://www.nature.com/naturegenetics/

1. Yachnis, A.T. \& Rorke, L.B. Neuropathology of Joubert syndrome. J. Child Neurol. 14, 655-659; discussion 669-672 (1999).

2. Quisling, R.G., Barkovich, A.J. \& Maria, B.L. Magnetic resonance imaging features and classification of central nervous system malformations in Joubert syndrome. J. Child Neurol. 14, 628-635; discussion 669-672 (1999).

3. Friede, R.L. \& Boltshauser, E. Uncommon syndromes of cerebellar vermis aplasia. I: Joubert syndrome. Dev. Med. Child Neurol. 20, 758-763 (1978).

4. Niesen, C.E. Malformations of the posterior fossa: current perspectives. Semin Pediatr. Neurol. 9, 320-334 (2002)

5. van Dorp, D.B., Palan, A., Kwee, M.L., Barth, P.G. \& van der Harten, J.J. Joubert syndrome: a clinical and pathological description of an affected male and a female fetus from the same sibship. Am. J. Med. Genet. 40, 100-104 (1991).

6. Kendall, B., Kingsley, D., Lambert, S.R., Taylor, D. \& Finn, P. Joubert syndrome: a clinico-radiological study. Neuroradiology 31, 502-506 (1990).

7. Joubert, M., Eisenring, J.J. \& Andermann, F. Familial dysgenesis of the vermis: a syndrome of hyperventilation, abnormal eye movements and retardation. Neurology 18 302-303 (1968).

8. Joubert, M., Eisenring, J.J., Robb, J.P. \& Andermann, F. Familial agenesis of the cerebellar vermis. A syndrome of episodic hyperpnea, abnormal eye movements, ataxia, and retardation. Neurology 19, 813-825 (1969).

9. Maria, B.L. et al. Molar tooth sign in Joubert syndrome: clinical, radiologic, and pathologic significance. J. Child Neurol. 14, 368-376 (1999).

10. Chance, P.F. et al. Clinical nosologic and genetic aspects of Joubert and related syndromes. J. Child Neurol. 14, 660-666; discussion 669-672 (1999)

11. Saraiva, J.M. \& Baraitser, M. Joubert syndrome: a review. Am. J. Med. Genet. 43 726-731 (1992)

12. Cantani, A., Lucenti, P., Ronzani, G.A. \& Santoro, C. Joubert syndrome. Review of the fifty-three cases so far published. Ann. Genet. 33, 96-98 (1990).

13. Ozonoff, S., Williams, B.J., Gale, S. \& Miller, J.N. Autism and autistic behavior in Joubert syndrome. J. Child Neurol. 14, 636-641 (1999).

14. Blair, I.P., Gibson, R.R., Bennett, C.L. \& Chance, P.F. Search for genes involved in Joubert syndrome: evidence that one or more major loci are yet to be identified and exclusion of candidate genes EN1, EN2, FGF8, and BARHL1. Am. J. Med. Genet. 107, 190-196 (2002)

15. Saar, K. et al. Homozygosity mapping in families with Joubert syndrome identifies a locus on chromosome 9q34.3 and evidence for genetic heterogeneity. Am. J. Hum. Genet. 65, 1666-1671 (1999).

16. Keeler, L.C. et al. Linkage analysis in families with Joubert syndrome plus oculo-rena involvement identifies the CORS2 locus on chromosome 11p12-q13.3. Am. J. Hum. Genet. 73, 656-662 (2003).

17. Valente, E.M. et al. Description, nomenclature, and mapping of a novel cerebello-renal syndrome with the molar tooth malformation. Am. J. Hum. Genet. 73, 663-670 (2003).

18. Lagier-Tourenne, C. et al. Homozygosity mapping of a third Joubert syndrome locus to 6q23. J. Med. Genet. 41, 273-277 (2004).

19. Jiang, X., Hanna, Z., Kaouass, M., Girard, L. \& Jolicoeur, P. Ahi-1, a novel gene encoding a modular protein with WD40-repeat and SH3 domains, is targeted by the Ahi-1 and Mis-2 provirus integrations. J. Virol. 76, 9046-9059 (2002).

20. Yang, Z. PAML: a program package for phylogenetic analysis by maximum likelihood. Comput. Appl. Biosci. 13, 555-556 (1997).

21. Yang, Z. Likelihood ratio tests for detecting positive selection and application to primate lysozyme evolution. Mol. Biol. Evol. 15, 568-573 (1998).

22. Watanabe, $\mathrm{H}$. et al. DNA sequence and comparative analysis of chimpanzee chromosome 22. Nature 429, 382-388 (2004).

23. Kullander, K. \& Klein, R. Mechanisms and functions of Eph and ephrin signalling. Nat. Rev. Mol. Cell Biol. 3, 475-486 (2002).

24. Couch, J. \& Condron, B. Axon guidance: Comm hither, Robo. Curr. Biol. 12, R741R742 (2002).

25. Altman, J. \& Bayer, S.A. Development of the Human Spinal Cord: An Interpretation Based on Experimental Studies in Animals (Oxford University Press, New York, 2001).

26. Maria, B.L., Boltshauser, E., Palmer, S.C. \& Tran, T.X. Clinical features and revised diagnostic criteria in Joubert syndrome. J. Child Neurol. 14, 583-590; discussion 590-581 (1999).

27. Merritt, L. Recognition of the clinical signs and symptoms of Joubert syndrome. Adv. Neonatal Care 3, 178-188 (2003).

28. Sobel, E. \& Lange, K. Descent graphs in pedigree analysis: applications to haplotyping, location scores, and marker-sharing statistics. Am. J. Hum. Genet. 58, 1323-1337 (1996).

29. Sobel, E., Sengul, H. \& Weeks, D.E. Multipoint estimation of identity-by-descent probabilities at arbitrary positions among marker loci on general pedigrees. Hum. Hered. 52, 121-131 (2001).

30. Cottingham, R.W. Jr., Idury, R.M. \& Schaffer, A.A. Faster sequential genetic linkage computations. Am. J. Hum. Genet. 53, 252-263 (1993). 